\title{
Polymer Chemistry

\section{Hyperbranched conjugated poly(tetraphenylethene): synthesis, aggregation-induced emission, fluorescent photopatterning, optical limiting and explosive detection $\dagger$}

\author{
Rongrong Hu, ${ }^{a b}$ Jacky W. Y. Lam, ${ }^{a b}$ Jianzhao Liu, ${ }^{a b}$ Herman H. Y. Sung, ${ }^{b}$ Ian D. Williams, ${ }^{b}$ Zhounan Yue, \\ Kam Sing Wong, ${ }^{c}$ Matthew M. F. Yuen ${ }^{d}$ and Ben Zhong Tang*abe
}

Received 4th February 2012, Accepted 19th March 2012

DOI: $10.1039 / \mathrm{c} 2 \mathrm{py} 20057 \mathrm{k}$

Tetraphenylethene-containing diyne (1), named 1,2-bis(4-ethynylphenyl)-1,2-diphenylethene, was synthesized and polymerized by $\mathrm{TaBr}_{5}$ catalyst, affording the hyperbranched polymer $h b$-P1 with a high molecular weight ( $M_{\mathrm{w}}$ up to 157800 ) in a nearly quantitative yield. The cyclotrimerization of 1(4-ethynylphenyl)-1,2,2-triphenylethene was also carried out to give 1,3,5- and 1,2,4-tris[4-(1,2,2triphenylvinyl)phenyl]benzene (1,3,5-2 and 1,2,4-2) that served as model compounds for structural characterization and properties investigation. The structures of $h b-\mathrm{P} \mathbf{1}$ and $\mathbf{2}$ are characterized by MS, IR, and NMR spectroscopies with satisfactory results. All the molecules and polymer are completely soluble in common organic solvents such as chloroform, toluene and THF. They show high thermal stability with degradation temperatures up to 443 and $446{ }^{\circ} \mathrm{C}$, respectively, in nitrogen and air. Whereas their solutions are almost non-emissive or weakly fluorescent, their aggregates in poor solvents and solid powders emit intensely with fluorescence quantum yields up to $81 \%$, demonstrating a novel phenomenon of aggregation-induced or enhanced emission. The unreacted triple bonds on the periphery of $h b$-P1 allow its thin film to cross-link under UV irradiation, generating well-resolved photopatterns. The hyperbranched polymer can limit harsh laser pulses. Its emission can be quenched efficiently by picric acid in solution and aggregated states with large quenching constants, suggesting that it is a promising fluorescent chemosensor for explosives detection.

\section{Introduction}

Hyperbranched polymers are a new class of macromolecule that show architectural beauty and multifaceted functionality of dendrimers. ${ }^{1}$ These highly branched macromolecules are readily prepared by one-pot, single-step polymerization procedures, accessible through different synthetic strategies. The most

${ }^{a}$ Fok Ying Tung Research Institute, The Hong Kong University of Science \& Technology (HKUST), Clear Water Bay, Kowloon, Hong Kong, China. E-mail: tangbenz@ust.hk

${ }^{b}$ Department of Chemistry and State Key Laboratory of Molecular Neuroscience, HKUST, Clear Water Bay, Kowloon, Hong Kong, China

'Department of Physics, HKUST, Clear Water Bay, Kowloon, Hong Kong, China

${ }^{d}$ Department of Mechanical Engineering, HKUST, Clear Water Bay, Kowloon, Hong Kong, China

${ }^{e}$ Department of Polymer Science and Engineering, Zhejiang University, Hang Zhou, China

$\dagger$ Electronic supplementary information (ESI) available: synthesis and characterization; crystal data and structure refinement for $Z-1$ and $E-\mathbf{1}$; IR, ${ }^{1} \mathrm{H}$ NMR and ${ }^{13} \mathrm{C}$ NMR spectra of 3 and 1,3,5-2; MS spectra of 1,3,5-2 and 1,2,4-2; GPC trace of $h b$-P1; photographs and emission spectra of 1,2,4-2 in THF-water mixtures. CCDC reference numbers 834091 and 834092 . For ESI and crystallographic data in CIF or other electronic format see DOI: $10.1039 / \mathrm{c} 2 \mathrm{py} 20057 \mathrm{k}$ commonly adopted approach is the self-condensation polymerization of $\mathrm{AB}_{n}$-type multifunctional monomers with $n \geq 2{ }^{2}$ These monomers, however, suffer from problems associated with the tendency of their mutually reactive A and $\mathrm{B}$ functional groups toward self-oligomerization. Because of the limited commercial availability and difficult synthetic access to multifunctional monomers bearing multiple, mutually reactive groups, alternative approaches such as the polymerization of $\mathrm{A}_{2}$ monomers with $\mathrm{B}_{n}$ monomers $(n \geq 3)$ have been developed. ${ }^{3}$ Other polymerization reactions, including self-condensing vinyl polymerizations initiated by cationic ${ }^{4}$ and radical catalysts ${ }^{5}$ and ring-opening multibranching polymerizations, ${ }^{6}$ have been explored, mainly for the synthesis of non-conjugated hyperbranched polymers. ${ }^{7}$ Hyperbranched macromolecules have been constructed from various functional groups, among which, the carbon-carbon triple-bond functionality uniquely stands out because it offers ready access to hyperbranched conjugated macromolecules. Being unsaturated, it accommodates various addition reactions, thus enabling the modification of the properties of polymers by post-manipulation.

Acetylene cyclotrimerization is a century-old reaction for the effective transformation of monoyne molecules to benzene rings. Polycyclotrimerizations of diyne molecules may result in the 
formation of hyperbranched polyarylenes. This $\mathrm{A}_{2}$ polycyclotrimerization approach will circumvent the synthetic difficulties encountered by the $\mathrm{AB}_{2}$ system and produce stable polymers consisting of robust benzene rings. Such a possibility has been actively explored in our laboratory. We have developed effective transition-metal catalyst systems for the polycyclotrimerization of diacetylenes or diynes (Scheme 1) ${ }^{8}$ Using this new polymerization technique, we prepared a variety of hyperbranched polyarylenes with different branching patterns, periphery archetypes, and core structures. The polymerization reactions proceeded rapidly under ambient conditions, giving polymeric products with high molecular weights (up to several million) in high yields (up to $100 \%$ ). The polymers were soluble in common organic solvents and the resultant solutions exhibited low intrinsic viscosity, thanks to the three-dimensional molecular architectures of the hyperbranched polyarylenes. Because aromatic rings were the basic constituent units, the polymers were thermally very stable, losing no weight below $\sim 500^{\circ} \mathrm{C}$ in the thermogravimetric analyses. The hyperbranched polyarylenes constructed from diyne monomers with conventional chromophores such as biphenyl and fluorene strongly attenuated optical pulses of powerful laser beams, whose optical limiting performance was superior to $\mathrm{C}_{60},{ }^{9}$ the best-known optical limiting material. ${ }^{10}$ They also emitted efficiently in the solution state. Their fluorescence quantum yields, however, became lower when fabricated into thin films, presumably due to the formation of aggregates, whose excited states often decay via non-radiative pathways.

In our search for efficient luminescent materials, we were attracted by a group of propeller-shaped molecules called siloles. These molecules were found to be virtually non-luminescent in solutions as isolated species but became highly emissive when aggregated as nanoparticles in poor solvents or fabricated into thin films in the solid state. The term of "aggregation-induced emission (AIE)" was coined for this extraordinary phenomenon because the silole molecules were induced to emit by aggregate formation. ${ }^{11}$ The silole derivative $\mathbf{4}(m)$ with ethynyl groups at the 2,5-positions could be cyclotrimerized in the presence of $\mathrm{TaBr}_{5}$, producing high molecular weight hyperbranched poly(2,5-silole)s $h b$-P4 $(m)$ in high yields. ${ }^{12}$ Similar to their monomers, the polymers were weakly fluorescent in solution but emitted intensely in the aggregated state. The silole-containing diynes, however, are difficult to prepare. ${ }^{12 b}$ It takes many steps of reactions to synthesize and the reactions involve reactive
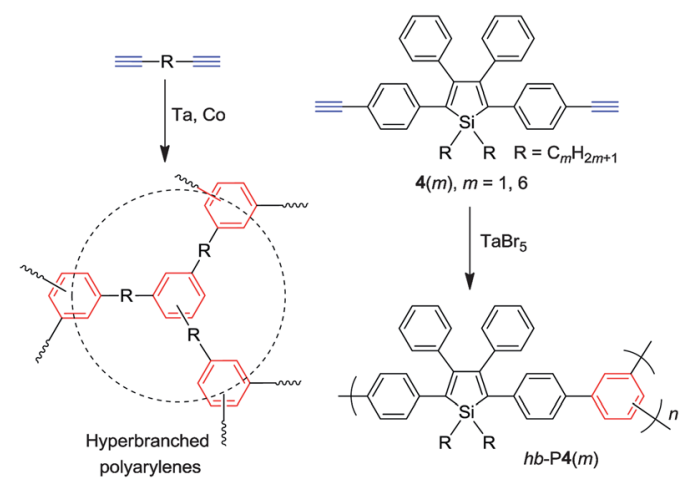

Scheme 1 Transition-metal catalyzed polycyclotrimerizations of diynes. organometallic species and sensitive intermediates. It would be nice if the polycyclotrimerization could be extended to other AIE-active diynes that can be readily prepared and modified through simple chemical reactions.

Tetraphenylethene (TPE) is another archetypal AIE luminogen and can be facilely prepared by a one-pot, functionalitytolerant coupling reaction. It enjoys high chemical stability, suffering no ring-opening reactions in basic medium as observed in siloles. It and its derivatives have found an array of high technological applications in optics, electronics, and environmental and biological sciences. ${ }^{13}$ If the diyne monomers containing TPE units can be polymerized, it will pave the way to the facile and economic synthesis of functional hyperbranched polymers. A TPE-containing diyne, however, possesses much less free volume than that with a silole unit, which makes it uncertain whether the resulting polymer will be soluble.

In this work, we explored the possibility of polycyclotrimerizing a TPE-functionalized diyne $\mathbf{1}$ (Scheme 2). In this paper, we show that monomer 1 can be effectively polycyclotrimerized by $\mathrm{TaBr}_{5}$ in toluene at room temperature, furnishing processable, high molecular weight hyperbranched poly(tetraphenylethene) $h b$-P1 in a high yield. The polymer is thermally stable, losing little of its weight at high temperatures. It undergoes a cross-linking reaction upon photoirradiation, which enables the generation of fluorescent photopatterns with good resolution. It can also function as a sensitive fluorescent chemosensor for the detection of explosives, with a superamplification effect observed in the emission quenching of the polymer nanoaggregates by picric acid.

\section{Experimental}

\section{Materials}

Tetrahydrofuran (THF) and toluene were distilled under normal pressure from sodium benzophenone ketyl under nitrogen immediately prior to use. Triethylamine was distilled and dried over potassium hydroxide. 4-Bromobenzophenone (5),

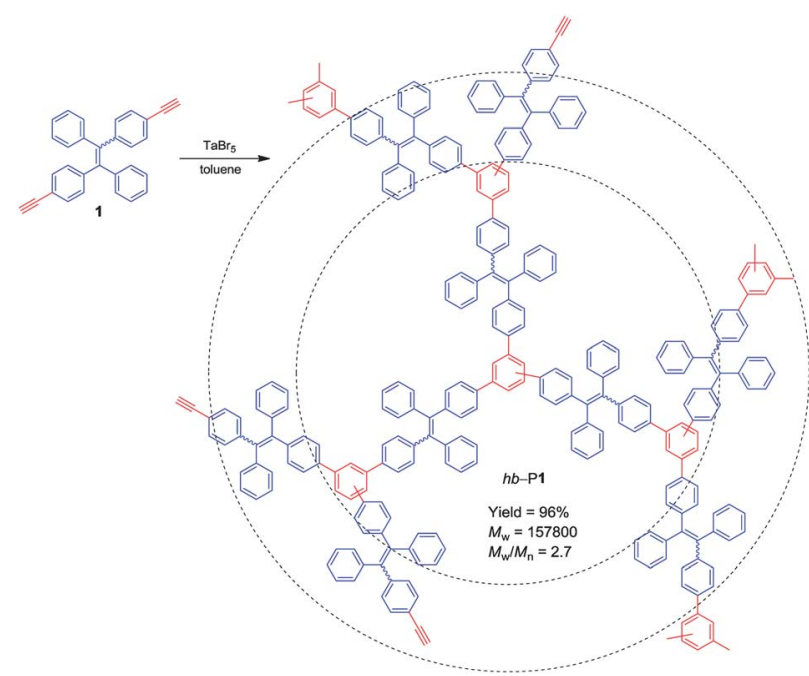

Scheme 2 Transformation of tetraphenylethene-containing diyne into a hyperbranched polymer via diyne polycyclotrimerization. 
diphenylmethane (7), n-butyllithium (n-BuLi), tantalum(v) bromide, tetraphenyltin, tantalum(v) chloride, dichlorobis(triphenylphosphine)palladium(II) $\quad\left[\mathrm{Pd}\left(\mathrm{PPh}_{3}\right)_{2} \mathrm{Cl}_{2}\right], \quad$ copper(I) iodide $(\mathrm{CuI})$, triphenylphosphine $\left(\mathrm{PPh}_{3}\right)$, zinc powder, titaniu$\mathrm{m}$ (Iv) chloride $\left(\mathrm{TiCl}_{4}\right)$, tetrabutylammonium fluoride (TBAF), $p$ toluenesulfonic acid (PTSA) and other chemicals and solvents were all purchased from Aldrich and used as received without further purification.

\section{Instruments}

${ }^{1} \mathrm{H}$ and ${ }^{13} \mathrm{C}$ NMR spectra were measured on Bruker ARX 300 NMR and Bruker ARX 400 NMR spectrometers using $\mathrm{CD}_{2} \mathrm{Cl}_{2}$ or $\mathrm{CDCl}_{3}$ as the deuterated solvent and tetramethylsilane (TMS; $\delta=0 \mathrm{ppm}$ ) as the internal standard. UV-vis absorption spectra were measured on a Milton Roy Spectronic 3000 array spectrophotometer. Photoluminescence (PL) spectra were recorded on a Perkin-Elmer spectrofluorometer LS 55. IR spectra were recorded on a Perkin-Elmer 16 PC FT-IR spectrophotometer. Thermogravimetric analysis (TGA) measurements were carried out under nitrogen or in air on a Perkin-Elmer TGA 7 analyzer at a heating rate of $10^{\circ} \mathrm{C} \mathrm{min}^{-1}$. Relative number $\left(M_{\mathrm{n}}\right)$ and weightaverage $\left(M_{\mathrm{w}}\right)$ molecular weights and polydispersity indices (PDI or $\left.M_{\mathrm{w}} / M_{\mathrm{n}}\right)$ of the polymers were estimated by a Waters Associates gel permeation chromatography (GPC) system equipped with RI and UV detectors. THF was used as the eluent at a flow rate of $1.0 \mathrm{~mL} \mathrm{~min}^{-1}$. A set of monodispersed linear polystyrenes covering the molecular weight range of $10^{3}-10^{7}$ was used as the standard for the molecular weight calibration. MALDI-TOF mass spectra were recorded on a GCT premier CAB048 mass spectrometer. Single crystal X-ray diffraction intensity data were collected at $100 \mathrm{~K}$ on a Bruker-Nonices Smart Apex CCD diffractometer with graphite monochromated Mo-K $\alpha$ radiation. Processing of the intensity data was carried out using the SAINT and SADABS routines and the structure and refinement were conducted using the SHELTL suite of X-ray programs (version 6.10). The cyclic voltammetry (CV) analysis was conducted on a CHI600A electrochemical workstation. All the measurements were carried out at room temperature using a conventional threeelectrode configuration. The working electrode was a glassy carbon electrode with a diameter of $2 \mathrm{~mm}$. The reference was a silver chloride electrode and the counter electrode was a platinum wire. The polymer solution was prepared in distilled dichloromethane. $0.1 \mathrm{M}$ of tetrabutylammonium hexafluorophosphate was used as the supporting electrolyte. The CV curves were measured at a scan rate of $100 \mathrm{mV} \mathrm{s}^{-1}$. Photocrosslinking reactions of the polymer films were conducted in air at room temperature using the $365 \mathrm{~nm}$ light obtained from a Spectroline ENF-280C/F UV lamp at a distance of $1 \mathrm{~cm}$ as the light source. The incident light intensity was $\sim 18.5 \mathrm{~mW} \mathrm{~cm}{ }^{-2}$. The film was prepared by spin-coating the polymer solution $(10 \%$ w/w in 1,2-dichloroethane) at $1500 \mathrm{rpm}$ for $1 \mathrm{~min}$ on a silicon wafer. The polymer film was dried in a vacuum oven at room temperature overnight. The photoresist patterns were generated using a copper photomask and taken on an optical microscope (Olympus B202) using a UV light source. The optical limiting experiments were carried out at $532 \mathrm{~nm}$, using $6 \mathrm{~ns}$ optical pulses generated from a frequency-doubled Q-switched Nd:YAG laser (Quanta Ray GCR-3) operating in a near Gaussian transverse mode with a repetition rate of $10 \mathrm{~Hz}$. The pulsed laser beam was focused onto a $1 \mathrm{~cm}$ quartz cell filled with a THF solution of the polymer. The incident and transmitted energies were measured by a Newport Power Meter (Model 1815-C) and every point of the optical limiting data was the average of at least 15 laser shots. The absolute fluorescence quantum yields of the solid powders were determined on a calibrated integrating sphere according to the method described by de Mello et al. ${ }^{14}$ A $325 \mathrm{~nm}$ CW light from a He-Cd laser was used for optical pumping. The studied sample was placed inside the integrating sphere, where the light emission was redistributed isotropically over the interior surface of the sphere. An optical fiber collected the light from the sphere and directed it to an Ocean Optics (Ocean Optics, Inc.) USB2000 miniature fiber optics spectrometer. The number of emitted photons was obtained by integrating the measured PL signal over the emission wavelengths. The response of the fiber and spectrometer detector system was normalized using a calibrated light source.

\section{Synthesis}

Compound 1,3,5-2, 1,2,4-2 and $h b$-P1 were prepared according to the synthetic routes shown in Scheme 2 and 3. Detailed procedures and characterization can be found in the supporting information. $\dagger$

\section{Preparation of aggregates}

Stock THF solutions of $\mathbf{2}$ and $h b$-P1 with a concentration of 0.1 $\mathrm{mM}$ were prepared. An aliquot $(1 \mathrm{~mL})$ of this stock solution was transferred to a $10 \mathrm{~mL}$ volumetric flask. After adding an appropriate amount of THF, water was added dropwise under vigorous stirring to furnish a $10 \mu \mathrm{M} \mathrm{THF}$-water mixture with a specific water fraction. The water content was varied in the range of $0-90 \mathrm{vol} \%$ (10 $\mathrm{mL}$ of $10 \mu \mathrm{M} \mathrm{THF}-$ water mixture with $95 \mathrm{vol} \%$ water content was prepared from $1 \mathrm{mM}$ of THF solution in the same manner). Absorption and emission spectra of the
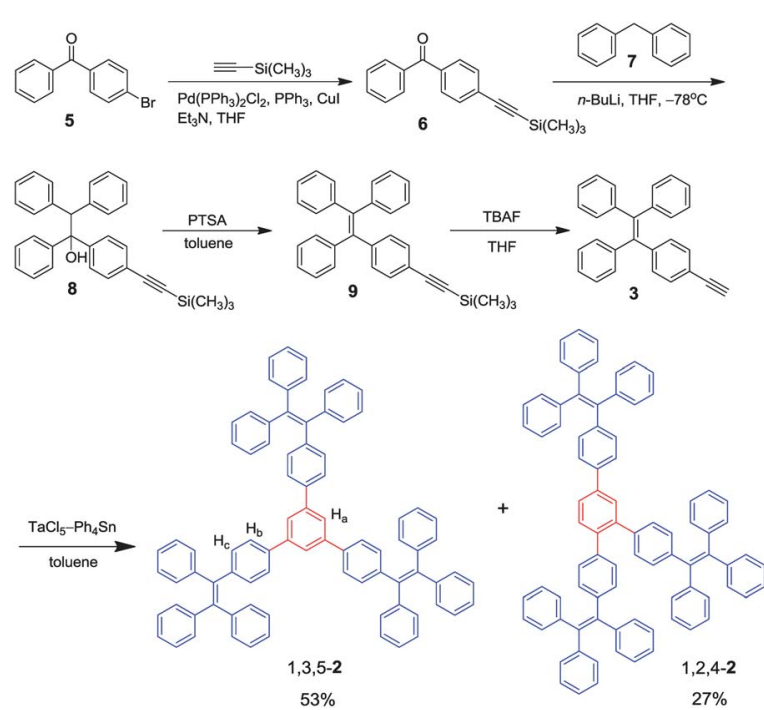

Scheme 3 Synthetic route for monoyne 3 and its transformation to trisubstituted benzenes. 
resulting solutions and aggregates were measured immediately after the sample preparation.

\section{Picric acid (PA) detection}

Stock solution of PA with a concentration of $2 \mathrm{mg} \mathrm{mL}^{-1}$ was prepared by dissolving appropriate amounts of PA in THF. PL titration was carried out by adding aliquots of PA solution into THF solution and THF-water mixtures with 50 and $90 \%$ water contents of $h b$-P1.

\section{Results and discussion}

\section{Monoyne model reaction}

Before studying the polycyclotrimerization of diyne 1, we synthesized a TPE-containing monoyne containing one triple bond (3) and utilized it as the monomer for the model reaction (Scheme 3). The palladium-catalyzed Sonogashira coupling reaction of 4-bromobenzophenone (5) with trimethylsilylacetylene gave compound $\mathbf{6}$, whose reaction with diphenylmethyllithium followed by dehydration generated 9 . The trimethylsilyl group in $\mathbf{9}$ was cleaved in the presence of TBAF, furnishing the desirable product $\mathbf{3}$ in a high yield. According to our previous study, monoynes, such as phenylacetylene, can be cyclotrimerized in the presence of $\mathrm{TaCl}_{5}-\mathrm{Ph}_{4} \mathrm{Sn}$ catalyst. ${ }^{9 c} \mathrm{We}$ thus tried to cyclotrimerize 3 using the same catalyst. After stirring 3 and $\mathrm{TaCl}_{5}-\mathrm{Ph}_{4} \mathrm{Sn}$ in toluene at room temperature for $16 \mathrm{~h}$, the solvent was evaporated and the crude product was purified by silica-gel column chromatography. Structural characterization by IR and NMR spectroscopies reveals that trisubstituted benzenes (2) are obtained, suggesting that $\mathbf{3}$ can undergo cyclotrimerization in the presence of $\mathrm{TaCl}_{5}-\mathrm{Ph}_{4} \mathrm{Sn}$. In contrast to our previous result, the 1,3,5-regiomer was obtained in a higher yield, presumably due to the steric effect of the bulky TPE unit.

\section{Diyne polycyclotrimerization}

After confirming that monoyne 3 can undergo cyclotrimerization, we then utilized the protocol to synthesize new hyperbranched polymers. We prepared TPE-containing diyne 1 according to the synthetic route shown in Scheme 4. The McMurry coupling reaction of compound $\mathbf{6}$ was promoted by $\mathrm{TiCl}_{4}$ and $\mathrm{Zn}$ in THF, giving a mixture of 1,2-bis(4-trimethylsilylphenyl)-1,2-diphenylethene (10) with a $Z / E$ ratio of $\sim 1: 1$. Desilylation of $\mathbf{1 0}$ in THF in the presence of tetrabutylammonium fluoride generated the desirable product 1 in $\sim 90 \%$ yield.

The polycyclotrimerization of $\mathbf{1}$ was catalyzed by $\mathrm{TaBr}_{5}$, instead of $\mathrm{TaCl}_{5}-\mathrm{Ph}_{4} \mathrm{Sn}$, in toluene under nitrogen at room temperature owing to its comparatively lower catalytic activity, which is less likely to induce uncontrollable propagation to give insoluble gels. ${ }^{8 c}$ The polymerization was also carried out at low

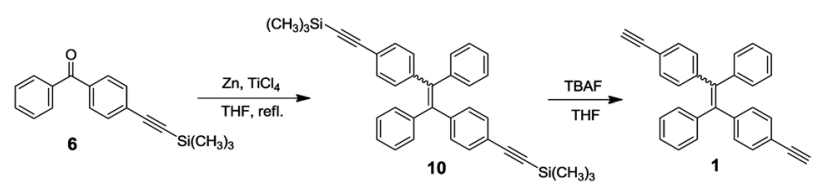

Scheme 4 Preparation of tetraphenylethene-containing diyne 1. monomer concentration for short reaction times $(9 \mathrm{~h})$ for the same reason mentioned above. The reaction was terminated by adding methanol and the mixture was then added into a large amount of methanol. The precipitates were collected, washed with methanol and dried in vacuum to a constant weight. Analysis by GPC shows an $M_{\mathrm{w}}$ value of 157800 (Fig. S1†), indicative of its polymeric nature. The polymer was obtained in a nearly quantitative yield $(\sim 96 \%)$ and was soluble in common organic solvents, enabling us to characterize its molecular structure by "wet" spectroscopic methods.

The $Z$ and $E$-isomers of monomer 1 cannot be separated by column chromatography due to their similar physical properties. However, two different types of crystal were isolated by the slow evaporation of a chloroform solution of $\mathbf{1}$. Analysis by singlecrystal X-ray crystallography shows that the $Z$-isomer crystallizes in a bulk-like fashion, while its $E$ counterpart forms needleshaped crystals. Fig. 1 shows their crystal structures and fluorescent photographs and Table $\mathrm{S} 1$ and $\mathrm{S} 2 \uparrow$ summarize their crystal data. The phenyl rings in both isomers are aligned almost perpendicularly to the central vinyl core. This imparts the molecules a twisted conformation, which hampers the close packing between the molecules. The crystals of the $Z$ - and $E$ isomers emit different colors upon photoexcitation. Whereas those of $Z$-1 show blue emission, the crystals of $E-1$ appear greenish-blue under UV light. The PL spectra shown in Fig. S2 $\uparrow$ support the visual observation described above. The PL spectrum of $E-1$ peaks at $484 \mathrm{~nm}$, which is $30 \mathrm{~nm}$ red-shifted from that of $Z \mathbf{- 1}$, presumably due to its higher conjugation.

\section{Structural characterization}

All the intermediates and products were characterized by standard spectroscopic methods and gave satisfactory analysis data corresponding to their expected molecular structures (see Electronic supplementary information for details $\dagger$ ).

The IR spectra of 3, 1,3,5-2 and 1,2,4-2 are given in Fig. S3. $\dagger$ The strong absorption bands observed at 3287 and $2109 \mathrm{~cm}^{-1}$ in 3 are associated with its $\equiv \mathrm{C}-\mathrm{H}$ and $\mathrm{C} \equiv \mathrm{C}$ stretching vibrations, respectively. These bands, however, are absent in the spectra of
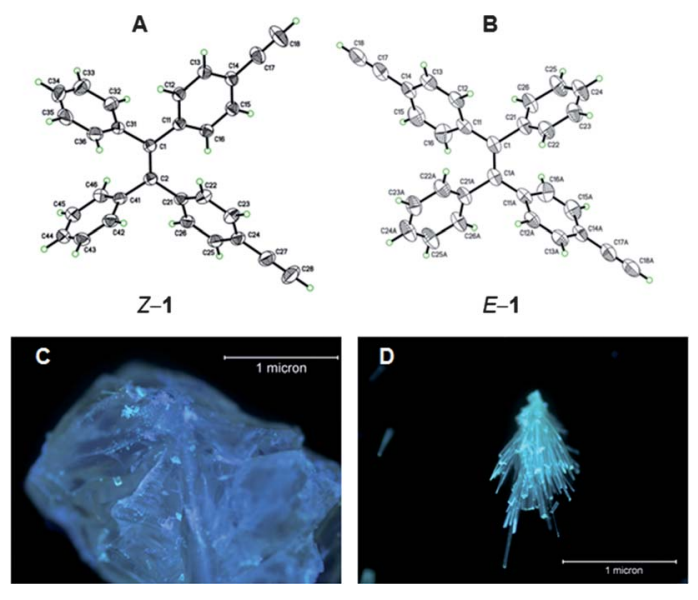

Fig. 1 Crystal structures and fluorescent images of (A and C) Z-1 (CCDC 834091) and (B and D) E-1 (CCDC 834092). The photographs were taken under a fluorescence microscope. 


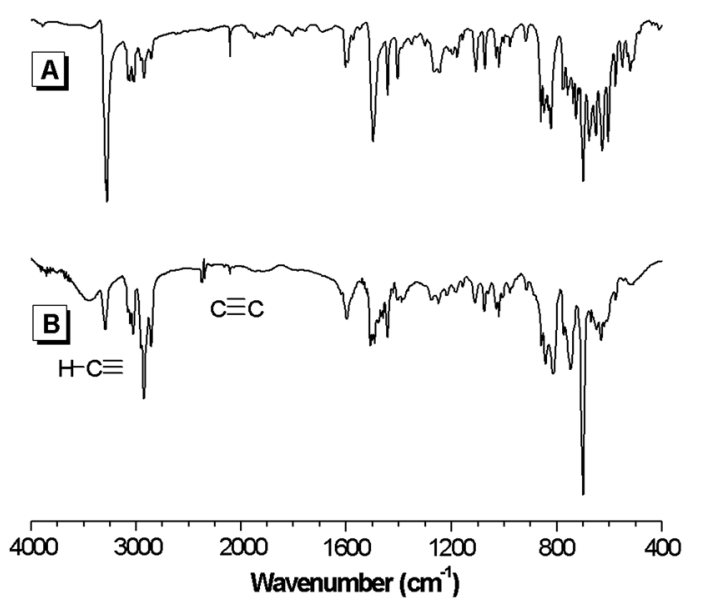

Fig. 2 IR spectra of (A) 1 and (B) $h b$-P1.

1,3,5-2 and 1,2,4-2, indicating all the triple bonds of $\mathbf{3}$ have been consumed by the cyclotrimerization reaction. The IR spectra of diyne $\mathbf{1}$ and its hyperbranched polymer $h b$-P1 are also provided in Fig. 2. The $\equiv \mathrm{C}-\mathrm{H}$ and $\mathrm{C} \equiv \mathrm{C}$ stretching vibrations of $\mathbf{1}$ occur at 3275 and $2106 \mathrm{~cm}^{-1}$, which become much weaker in the spectrum of $h b$-P1. Meanwhile, the absorption bands associated with aromatic $\mathrm{C}-\mathrm{H}$ stretching vibrations are intensified after the polymerization, suggesting that most of the triple bonds of $\mathbf{1}$ have been converted to benzene rings of $h b$-P1 by the polycyclotrimerization reaction.

Similar results are obtained from the NMR analysis. Fig. S4 $\dagger$ shows the ${ }^{1} \mathrm{H}$ NMR spectra of $\mathbf{3}$ and 1,3,5-2 in dichloromethane$d_{2}$ as an example. The spectrum of 1,3,5-2 displays no acetylene proton resonance of $\mathbf{3}$ at $\delta 3.07$, indicative of the complete consumption of the triple bond by the cyclotrimerization reaction. Because of its $\mathrm{C}_{3}$-symmetrical structure, the ${ }^{1} \mathrm{H}$ NMR spectrum of 1,3,5-2 is more simple and better-resolved than that of 1,2,4-2. Compared to 3 , the spectrum of 1,3,5-2 displays a new singlet peak at $\delta 7.63$ associated with the proton resonance of the newly formed benzene ring $\left(\mathrm{H}_{\mathrm{a}}\right)$. The peak at $\delta 7.39 \mathrm{ppm}$, on the other hand, originates from the absorptions of the aromatic protons $\left(\mathrm{H}_{\mathrm{b}}\right)$ next to $\mathrm{H}_{\mathrm{a}}$. The rest of the protons resonate at $\sim \delta 7.03$. No unexpected signals are observed, suggesting that the product is indeed 1,3,5-2 with the molecular structure shown in Scheme 3.

The ${ }^{13} \mathrm{C}$ NMR spectra of $\mathbf{3}$ and 1,3,5-2 are shown in Fig. S5. $\dagger$ The acetylene carbon atoms of $\mathbf{3}$ resonate at $\delta 76.8$ and 83.1, which disappear completely in the spectrum of 1,3,5-2. New peaks emerge at the aromatic absorption region due to the formation of a new benzene ring by the cyclotrimerization reaction. The structures of 1,3,5-2 and 1,2,4-2 are further characterized by mass spectroscopy (Fig. S6 and S7 $\dagger$ ). The spectra give $\mathrm{M}^{+}$peaks at 1068.339 for $1,3,5-2$ and 1068.579 for $1,2,4-2$, which fit well with the calculated value of 1068.470.

Fig. 3 shows the ${ }^{1} \mathrm{H}$ NMR spectra of $\mathbf{1}$ and $h b$-P1 in dichloromethane- $d_{2}$. The peak at $\delta 3.09$ assigned to the acetylene proton resonance of diyne $\mathbf{1}$ is substantially weakened in the spectrum of $h b-\mathrm{P} \mathbf{1}$, due to the conversion of the triple bonds of $\mathbf{1}$ into benzene rings of $h b$-P1 by the polymerization. The absorption peaks in the aromatic region of $\mathbf{1}$ are significantly broadened and emerged after the polymerization due to the rigid and

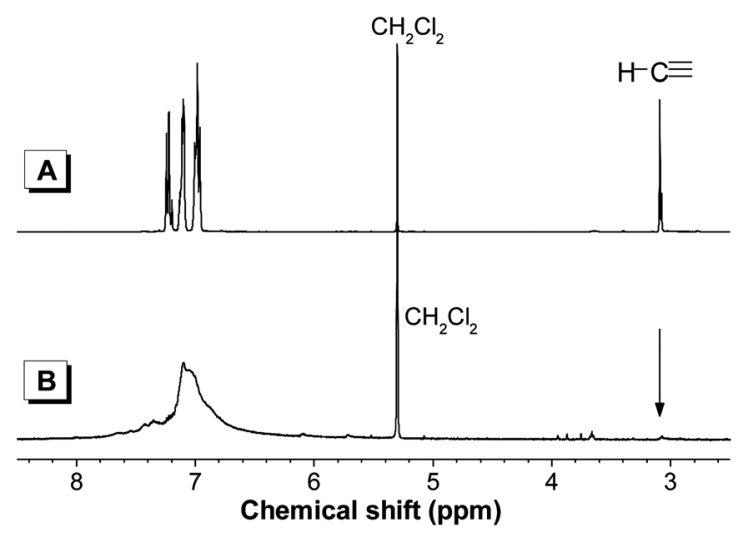

Fig. $3{ }^{1} \mathrm{H}$ NMR spectra of (A) 1 and (B) $h b$-P1 in dichloromethane- $d_{2}$.

irregular structure of the polymer, which possesses a random mixture of 1,3,5- and 1,2,4-trisubstituted benzenes. This also hampers the calculation of the degree of branching of the polymer.

\section{Solubility and stability}

Despite their conjugated aromatic structures without multiple substituents, 1,3,5-2, 1,2,4-2 and $h b$-P1 possess good solubility in common organic solvents, such as toluene, dichloromethane, chloroform, and THF. This may be due to the twisted conformation of the TPE unit, which results in large intermolecular distances and hence creates free volume to interact with the solvent molecules. Polymer $h b$-P1 also possesses good filmforming ability and can form tough thin films by spin-coating or solution-casting of its solution.

Since 1,3,5-2, 1,2,4-2 and $h b$-P1 are constructed from aromatic rings, they are anticipated to show high thermal stability. ${ }^{15}$ The unreacted triple bonds at the periphery of $h b$-P1 may undergo thermally-induced polymerization at high temperatures, generating a crosslinked product with high resistance to thermolytic attack. As shown in Fig. 4, both $\mathbf{2}$ and $h b$-P1 are thermally very stable, showing degradation temperatures $\left(T_{\mathrm{d}}{ }^{\prime} \mathrm{s}\right)$ at $428-463{ }^{\circ} \mathrm{C}$

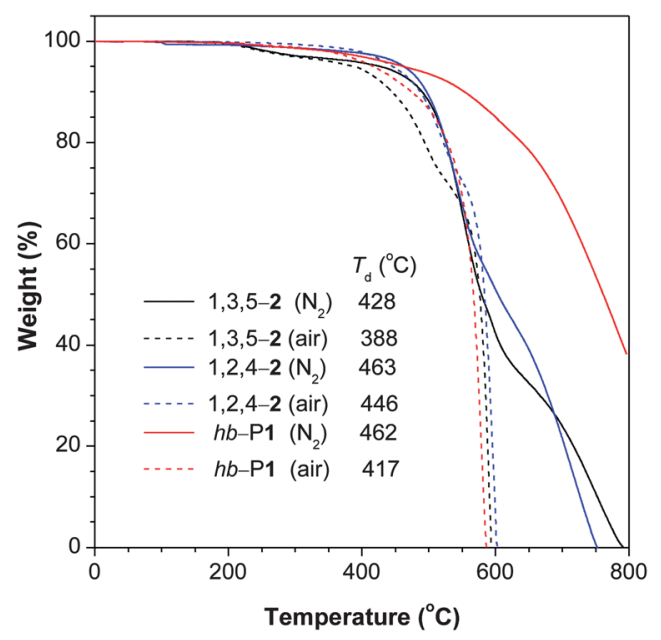

Fig. 4 TGA thermograms of 1,3,5-2, 1,2,4-2 and $h b$-P1 recorded under nitrogen and in air at a heating rate of $10{ }^{\circ} \mathrm{C} \mathrm{min}^{-1}$. 
under nitrogen and $388-446{ }^{\circ} \mathrm{C}$ in air. More than $80 \%$ of their weight is retained when they are heated to $500{ }^{\circ} \mathrm{C}$ under nitrogen. Even at $760{ }^{\circ} \mathrm{C}, h b$-P1 pyrolyses in a high yield $(>50 \%)$, indicating its strong resistance to thermolysis.

\section{Photophysical properties}

Fig. 5 shows the absorption spectra of 1,3,5-2, 1,2,4-2 and $h b$-P1 in THF solutions with a concentration of $10 \mu \mathrm{M}$. The absorption spectra of 1,3,5-2 and 1,2,4-2 both peak at about $326 \mathrm{~nm}$, suggesting that the substitution position exerts little influence on the electronic conjugation of the molecule. The absorption maximum of $h b-\mathrm{P} 1$ is located at $335 \mathrm{~nm}$, which is slightly redshifted from those of $1,3,5-2$ and 1,2,4-2, indicating that the polymer possesses a higher conjugation.

The impression of the AIE features of 1,3,5-2 and $h b$-P1 can be reflected by the fluorescent photographs taken in THF and THF-water mixtures. The THF solution of 1,3,5-2, emits no light under UV irradiation (Fig. 6A). Addition of water, a nonsolvent for $\mathbf{2}$, into the solution induces its molecules to aggregate and enhances its emission intensity. The emission remains low at water contents lower than $60 \mathrm{vol} \%$. Afterwards, the emission starts to increase swiftly. The higher the water content, the stronger the light emission. A similar phenomenon was also observed in 1,2,4-2 (Fig. S8†). In contrast to 1,3,5-2, $h b-\mathrm{P} \mathbf{1}$ is somewhat emissive in THF although the intensity is weak (Fig. 7A). The addition of water into the THF solution aggregates the polymer chains and consequently increases the light emission.

In addition to the visual observations, we examined the emission behaviors of $1,3,5-2$ and $h b$-P1 using a PL spectrophotometer. As depicted in Fig. 6B and 6C, the PL spectrum of $1,3,5-2$ in THF is basically a flat line parallel to the abscissa. When a large amount of water $(>60 \mathrm{vol} \%)$ is added to the THF solution, an emission peak emerges at $488 \mathrm{~nm}$. From the isolated species in THF solution to the aggregates in $90 \%$ aqueous mixture, the PL intensity rises by 933 -fold. The pure THF solution of $h b-\mathrm{P} \mathbf{1}$ does emit faintly at $501 \mathrm{~nm}$, which is slightly red-shifted from that of $1,3,5-2$. In the presence of a small

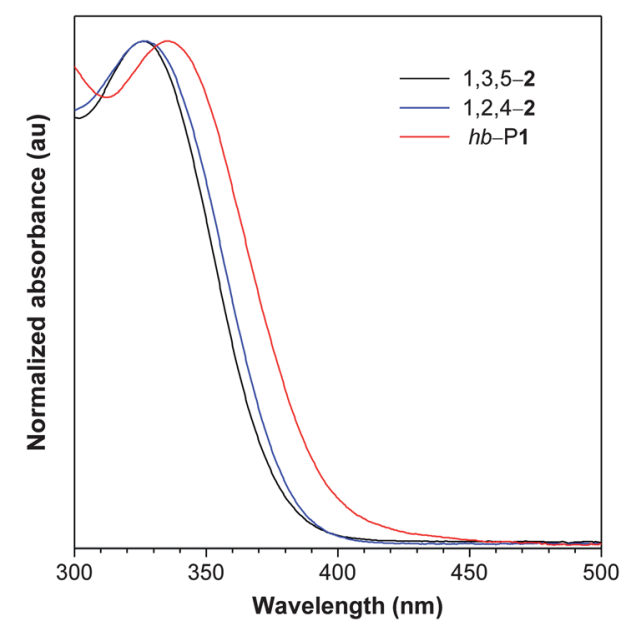

Fig. 5 Normalized absorption spectra of 1,3,5-2, 1,2,4-2 and $h b$-P1 in THF solutions. Concentration: $10 \mu \mathrm{M}$.

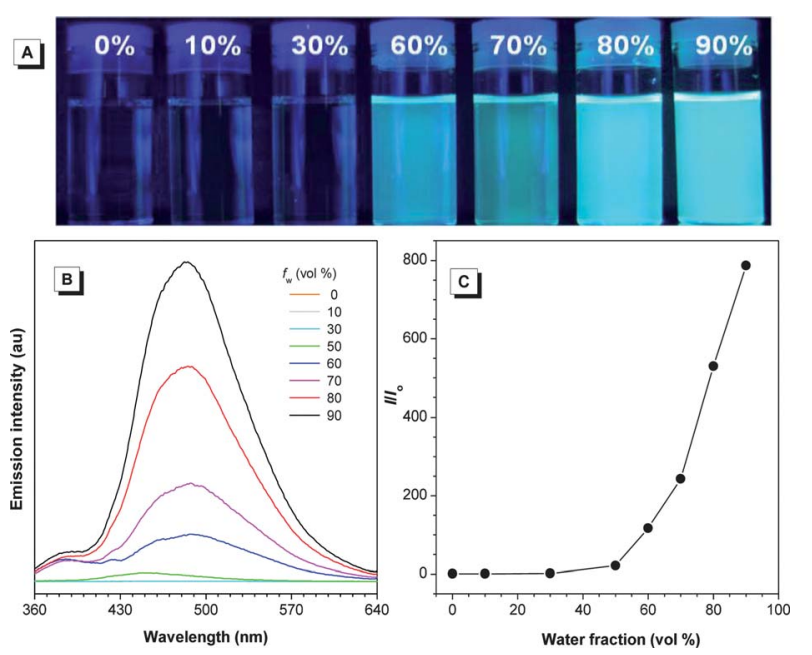

Fig. 6 (A) Photographs of 1,3,5-2 in THF-water mixtures with different water fractions $\left(f_{\mathrm{w}}\right)$ taken under $365 \mathrm{~nm}$ UV irradiation from a hand-held UV lamp. (B) Emission spectra of 1,3,5-2 in THF-water mixtures. (C) Plot of $\left(I / I_{0}\right)$ values versus the compositions of the aqueous mixtures. Solution concentration: $10 \mu \mathrm{M}$; excitation wavelength: $326 \mathrm{~nm}$.

amount of water, the emission becomes stronger subsequently and keeps rising with increasing water content. At $95 \%$ water content, the PL intensity increases by 11 -fold without a noticeable shift in the emission maximum. Clearly, $h b-\mathrm{P} \mathbf{1}$ is more sensitive to the changes in its surrounding environment than its low molecular weight counterparts. Even at low water content, the subtle change in the solvating power of the solvent mixture can cause the polymer strands to aggregate and hence change the emission behavior.

We also measured the fluorescence quantum yield $\left(\Phi_{\mathrm{F}}\right)$ of the molecules and the polymer in the solution and aggregated states and the results are summarized in Table 1 . The $\Phi_{\mathrm{F}}$ value of 1,3,52 in the THF-water mixture (64.1\%) is much higher than that in

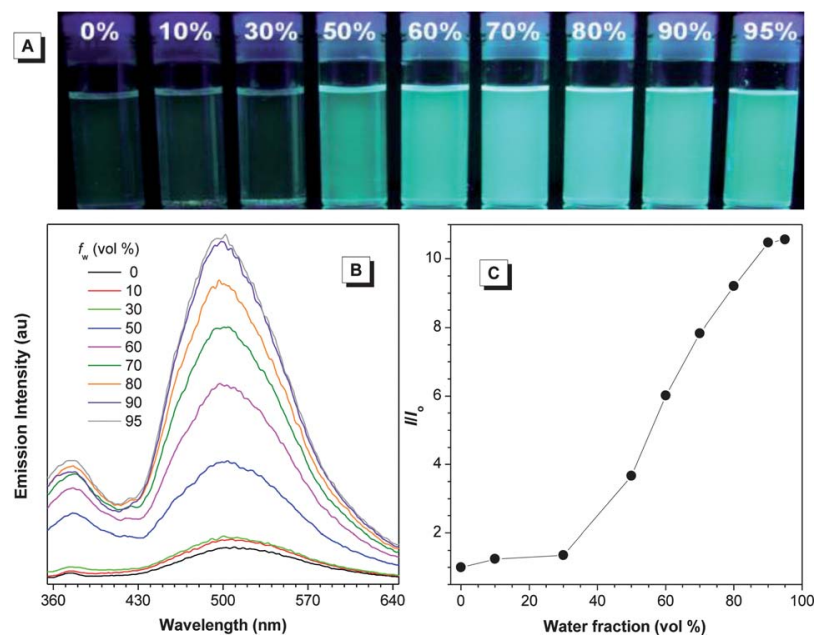

Fig. 7 (A) Photographs of $h b$-P1 in THF-water mixtures with different water fractions $\left(f_{\mathrm{w}}\right)$ taken under $365 \mathrm{~nm}$ UV irradiation from a hand-held UV lamp. (B) Emission spectra of $h b$-P1 in THF-water mixtures. (C) Plot of $\left(I / I_{0}\right)$ values versus the compositions of the aqueous mixtures. Solution concentration: $10 \mu \mathrm{M}$; excitation wavelength: $335 \mathrm{~nm}$. 
Table 1 Optical properties of 1,3,5-2, 1,2,4-2, and $h b-\mathrm{P} \mathbf{1}^{a}$

\begin{tabular}{llllllr}
\hline Compd & $\lambda_{\mathrm{ab}} / \mathrm{nm}$ & $\lambda_{\mathrm{em}} / \mathrm{nm}$ & $\begin{array}{l}\Phi_{\text {Soln }} \\
(\%)\end{array}$ & $\begin{array}{l}\Phi_{\text {Aggre }} \\
(\%)\end{array}$ & $\begin{array}{l}\Phi_{\text {Solid }} \\
(\%)\end{array}$ & $\alpha_{\text {AIE }}$ \\
\hline $1,3,5-2$ & 326 & 488 & 0.31 & 64.1 & 81 & 207 \\
$1,2,4-2$ & 327 & 488 & 0.26 & 38.0 & 67 & 146 \\
$h b-\mathbf{P 1}$ & 335 & 501 & 3.05 & 45.4 & 47 & 15
\end{tabular}

${ }^{a}$ Abbreviations: $\lambda_{\mathrm{ab}}=$ absorption maximum in THF solution $(10 \mu \mathrm{M})$, $\lambda_{\mathrm{em}}=$ emission maximum in $\mathrm{THF}-\mathrm{H}_{2} \mathrm{O}$ mixture $(1: 9 \mathrm{v} / \mathrm{v}), \Phi_{\text {Soln }}$ and $\Phi_{\text {Aggre }}=$ fluorescence quantum yields in THF and THF- $\mathrm{H}_{2} \mathrm{O}$ mixture $(1 / 9 \mathrm{v} / \mathrm{v})$ estimated using fluorescein $\left(\Phi_{\mathrm{F}}=97 \%\right.$ in $\left.0.1 \mathrm{M} \mathrm{NaOH}\right)$ as standard, $\alpha_{\mathrm{AIE}}=\Phi_{\mathrm{Aggre}} / \Phi_{\text {Soln }}, \Phi_{\text {Solid }}=$ fluorescent quantum yield of solid powder determined by a calibrated integrating sphere.

THF $(0.31 \%)$, giving a corresponding $\alpha_{\mathrm{AIE}}$ value of 207 , where $\alpha_{\mathrm{AIE}}$ is the AIE factor defined by the following equation: $\alpha_{\mathrm{AIE}}=$ $\Phi_{\mathrm{F}_{\text {aggr }}} / \Phi_{\mathrm{F}_{\text {soln }}}$. The $\alpha_{\mathrm{AIE}}$ value of 1,3,5-2 is larger than that of TPE (61). This may be due to the presence of more rotatable phenyl rings in 1,3,5-2, whose motions consume the energy of the excitons via a nonradiative relaxation channel and hence decreases the $\Phi_{\mathrm{F}}$ value in the solution state dramatically. 1,2,4-2 exhibits a similarly low $\Phi_{\mathrm{F}}$ value $(0.26 \%)$ in THF but a much higher value $(38.0 \%)$ in the aggregated state. Compared with 1,3,5-2 and $1,2,4-2, h b$-P1 is more emissive in THF solution $\left(\Phi_{\mathrm{F}}=3.05 \%\right)$, which is 10 -fold higher than that of $1,3,5-2$. Its $\Phi_{\mathrm{F}}$ value is boosted to $45.4 \%$ in $90 \%$ aqueous mixture, showing the phenomenon of aggregation-enhanced emission (AEE). ${ }^{16}$ The absolute $\Phi_{\mathrm{F}}$ values of solid powders of $\mathbf{2}$ and $h b$-P1 were also determined by a calibrated integrating sphere. While 1,3,5-2 and 1,2,4-2 exhibit reasonably high $\Phi_{\text {Solid }}$ values (81 and 67\%), that of $h b-\mathrm{P} 1$ is $47 \%$. As discussed before, the phenyl rings of the TPE units of 1,3,5-2 and 1,2,4-2 undergo free rotation in THF, which weakens the light emission and results in a lower $\Phi_{\mathrm{F}}$ value and a stronger AIE effect. The TPE units in $h b$-P1 are knitted together by benzene rings, which have partially limited their intramolecular rotations. The energy loss via nonradiative rotational channels is greatly reduced, thus making $h b$-P1 weakly emissive in the solution state. The intramolecular rotations are further restricted in the aggregated state, thus leading to the further enhancement of light emission.

\section{Redox activity}

The electrochemical properties of 1,3,5-2 and $h b$-P1 were studied by cyclic voltammetry in dry dichloromethane containing $0.1 \mathrm{M}$ of tetrabutylammonium hexafluorophosphate. The potential was linearly scanned between -1.0 and $1.6 \mathrm{~V}$ at a scan rate of $100 \mathrm{mV}$

Table 2 Electrochemical properties of 1,3,5-2 and $h b-\mathrm{P1}^{a}$

\begin{tabular}{llllll}
\hline Compd & $\Phi_{\mathrm{p}} / \mathrm{V}$ & $\mathrm{HOMO} / \mathrm{eV}$ & $\lambda_{\text {onset }} / \mathrm{nm}$ & $E_{\mathrm{g}} / \mathrm{eV}$ & $\mathrm{LUMO} / \mathrm{eV}$ \\
\hline $1,3,5-\mathbf{2}$ & 0.74 & -5.18 & 381 & 3.26 & -1.92 \\
$h b-\mathrm{P1}$ & 1.00 & -5.44 & 416 & 2.98 & -2.46
\end{tabular}

${ }^{a}$ Abbreviations: $\Phi_{\mathrm{p}}=$ oxidation potential determined from the onset of the first oxidation wave, HOMO = highest occupied molecular orbital calculated using the equation: $\mathrm{HOMO}=-4.44-\Phi_{\mathrm{p}} \mathrm{eV}, \lambda_{\text {onset }}=$ onset wavelength determined from the absorption spectrum, $E_{\mathrm{g}}=$ energy band gap determined from the onset absorption wavelength, LUMO = lowest unoccupied molecular orbital calculated from the equation: $\mathrm{LUMO}=E_{\mathrm{g}}+$ HOMO $\mathrm{s}^{-1}$. The voltammograms of 1,3,5-2 and $h b$-P1 exhibit anodic waves associated with the oxidation of the conjugated hydrocarbon at $\sim 0.7$ and $1.0 \mathrm{~V}$, from which HOMO energies of -5.18 and $-5.44 \mathrm{eV}$ are calculated, respectively (Table 2 ). The energy band gap can be determined from the onset absorption wavelength and is equal to $3.26 \mathrm{eV}$ for $1,3,5-2$ and $2.98 \mathrm{eV}$ for $h b$-P1, nicely explaining the bathochromic shift in the absorption and emission in $h b$-P1.

\section{Photo-crosslinking and photopatterning}

Since $h b$-P1 is emissive in the solid state and possesses many cross-linkable triple bonds on its periphery, we explored its potential use as a fluorescent imaging and sensitive photoresist material. The UV irradiation of a thin-film of $h b$-P1 through a negative copper photomask readily crosslinks the exposed regions, whereas the unexposed regions are removed by development in 1,2-dichloroethane. A negative photoresist pattern with sharp edges is thus generated (Fig. 8). The pattern can be clearly seen under daylight and emits a strong blue light under UV illumination.

\section{Nonlinear optical properties}

Lasers are widely used in many applications and pose a potential hazard to eyes and cause damage to human tissue and even death. ${ }^{17}$ Thus, scientists have fabricated optical limiting devices, which can offer high transmittance at low inputs but low transmittance at high laser doses. Since molecules with extended conjugated systems may show optical limiting properties, ${ }^{18}$ we thus checked whether our thermally stable hyperbranched polymer would act as an optical limiter. As shown in Fig. 9, the THF solution of $h b$-P1 attenuates the power of intense $532 \mathrm{~nm}$ optical pulses. The transmitted fluence increases initially with the incident fluence but starts to deviate from linearity when the incident fluence exceeds $60 \mathrm{~J} \mathrm{~cm}^{-2}$. The optical limiting properties, coupled with its high thermal stability and good processability, make $h b$-P1 a promising candidate material for high-tech applications.
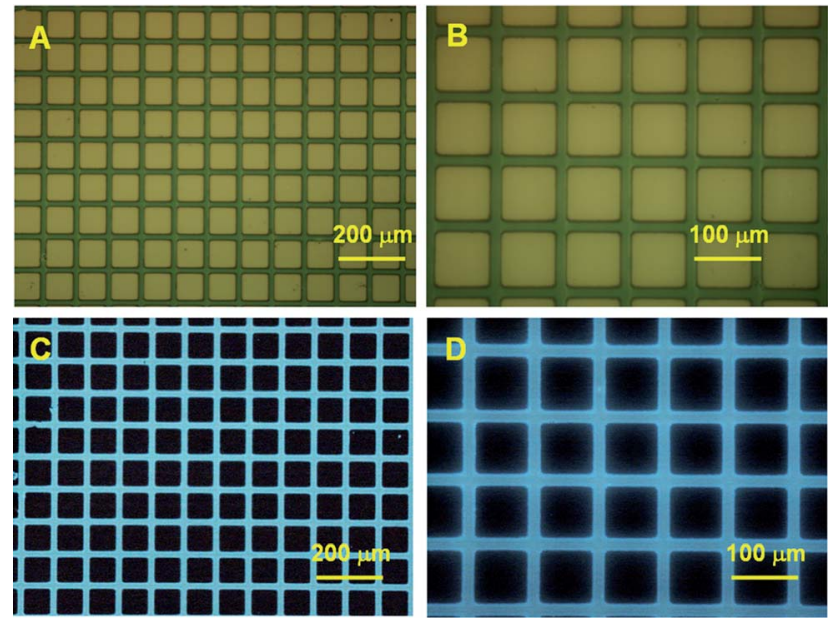

Fig. 8 Negative photoresist patterns generated by the photolithography of $h b$-P1 taken under (A and B) daylight and (C and D) UV light illumination. 


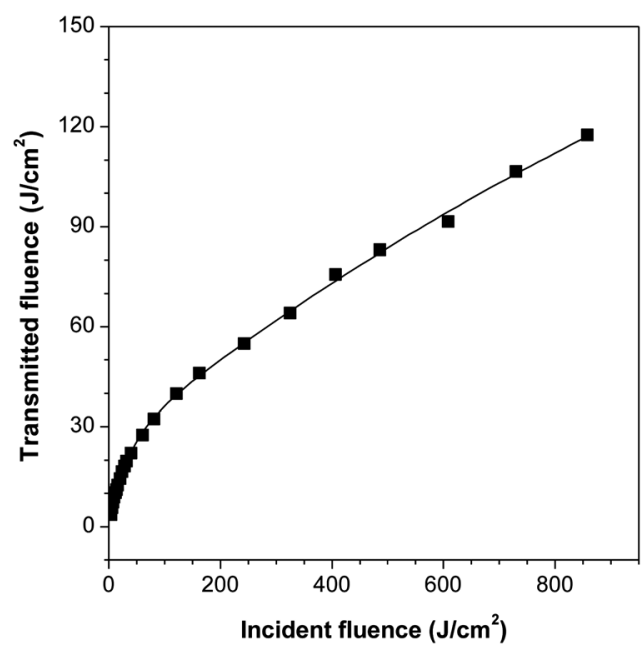

Fig. 9 Optical limiting responses to $6 \mathrm{~ns}, 532 \mathrm{~nm}$ optical pulses, of THF solution of $h b-\mathrm{P} 1$. Solution concentration: $0.02 \mathrm{mg} \mathrm{mL}^{-1}$.

\section{Explosive detection}

Conjugated polymers are promising materials for fluorescent chemosensors due to their amplified response and superior sensitivity to analytes, in comparison to their low molar mass congeners. ${ }^{19}$ In particular, those constructed from polymers with AIE characteristics are the most intriguing because they suffer no emission quenching problems caused by intrinsic autoaggregation of polymer chains and analyte-induced aggregation. Nitroaromatics such as 2,4,6-trinitrotoluene (TNT) and 2,4dinitrotoluene (DNT) are warfare explosives, the detection of which has antiterrorism implications. ${ }^{20}$ Since TNT and DNT are not commercial available, picric acid (PA) is used as a model explosive in this work. It is anticipated that TNT and DNT exert a similar effect as PA on the light emission of the polymer.

The PL of $h b$-P1 is progressively weakened when an increasing amount of PA is added into its solution in THF or its nanoaggregates in aqueous mixture (Fig. 10). The PL quenching can be detected at a PA concentration of $1 \mathrm{ppm}$. The $\mathrm{PL}$ of the polymer aggregates suspended in aqueous mixtures decreases at a much faster rate than their isolated species dissolved in THF. At a PA concentration of $0.70 \mathrm{mM}$, no light was emitted from the THF solution. On the contrary, the PL of the polymer aggregates in 50 and $90 \%$ aqueous mixtures is quenched completely at a lower PA concentration of $0.52 \mathrm{mM}$, indicative of their higher sensitivity to the presence of PA. The Stern-Volmer plots of relative PL intensity $\left(I_{0} / I\right)$ versus $\mathrm{PA}$ concentration in THF solution as well as the nanoaggregates suspended in aqueous mixtures give upward bending curves, instead of straight lines (Fig. 10D), revealing that the PL quenching becomes more efficient with increasing quencher concentration. A similar phenomenon was also observed in our previous study ${ }^{19 c, 19 i}$ and the PL annihilation is caused by static quenching, in which the electron-rich polymer strands bind to the electron-deficient quencher molecules and are in the nonemissive or dark state. Large quenching constants up to $429000 \mathrm{M}^{-1}$ are deduced from the plot, which are much higher than those of polysiloles and polygermoles reported previously $\left(6710-11000 \mathrm{M}^{-1}\right){ }^{21}$
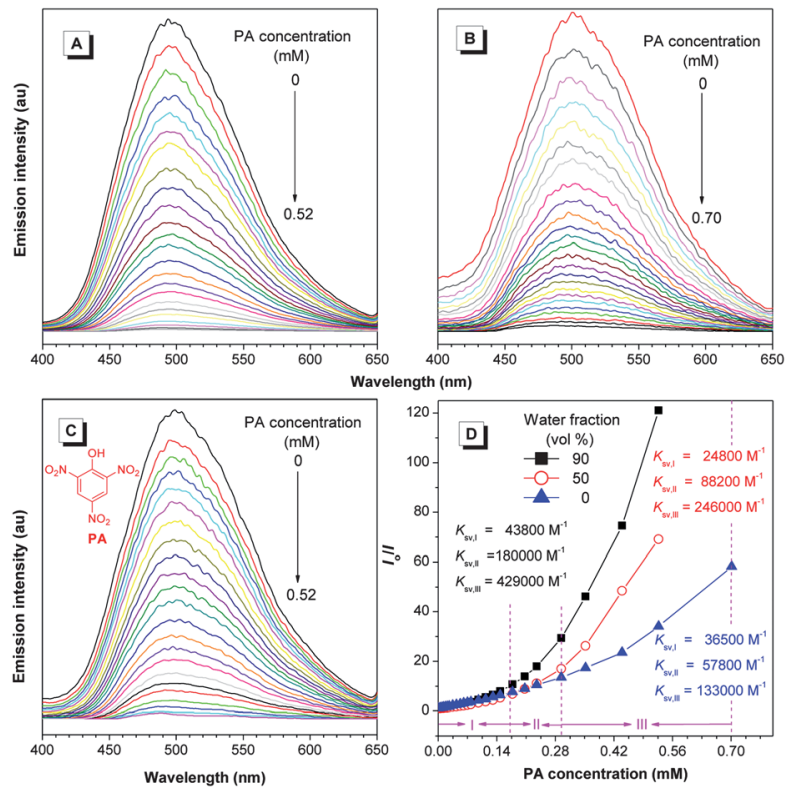

Fig. 10 PL spectra of $h b$-P1 with different PA concentrations in (A) THF-water mixture with $90 \%$ water fraction, (B) THF solution, and (C) THF-water mixture with $50 \%$ water fraction. Solution concentration: $10 \mu \mathrm{M}$; excitation wavelength: $335 \mathrm{~nm}$. (D) Stern-Volmer plots of $\left(I_{0} / I\right)$ value versus $[\mathrm{PA}]$ in THF and THF-water mixtures with 50 and $90 \%$ water contents. $I_{0}=$ intensity at $[\mathrm{PA}]=0 \mathrm{mM}$.

\section{Conclusions}

In this work, hyperbranched polyphenylene was synthesized in a high yield with a high molecular weight by the polycyclotrimerization of a tetraphenylethene-containing diyne catalyzed by $\mathrm{TaBr}_{5}$. Under similar conditions, 1-(4-ethynylphenyl)-1,2,2-triphenylethene was converted into 1,3,5- and 1,2,4-trisubstituted benzenes that serve as model compounds for structural characterization and properties investigations. All the molecules and polymer are characterized by standard spectroscopic methods with satisfactory results. They are thermally stable with high degradation temperatures under nitrogen and in air. Whereas they are nonemissive or weakly fluorescent in solutions, they become strong emitters when aggregated in poor solvents and in the solid state, demonstrating the phenomenon of aggregation-induced or enhanced emission. The hyperbranched polymer undergoes a crosslinking reaction readily upon UV irradiation, generating fluorescent photopatterns with good resolution. It exhibits nonlinear optical properties and can limit harsh laser pulses. The emission of the polymer in the solution and aggregated states can be quenched efficiently by picric acid with large quenching constants, suggesting that it is a promising fluorescent chemosensor for explosive detection. Materials with such attributes are anticipated to find an array of high-tech applications.

\section{Acknowledgements}

The work reported in this paper was partially supported by the National Science Foundation of China (20974028), the RPC and SRFI Grants of HKUST (RPC10SC13, RPC11SC09, and SRFI11SC03PG), the Research Grants Council of Hong Kong 
(604711, 603509, HKUST2/CRF/10, and N_HKUST620/11), the Innovation and Technology Commission (ITCPD/17-9) and the University Grants Committee of Hong Kong (AoE/P-03/08 and T23-713/11-1). B.Z.T. thanks the support from the Cao Guangbiao Foundation of Zhejiang University.

\section{Notes and references}

1 (a) A. Hult, M. Johansson and E. Malmström, Adv. Polym. Sci., 1999, 143, 1-34; (b) S. M. Grayson and J. M. J. Frechet, Chem. Rev., 2001, 101, 3819-3867.

2 P. J. Flory, J. Am. Chem. Soc., 1952, 74, 2718-2723.

3 (a) F. Wang, M. S. Wilson, R. D. Rauh, P. Schottland and J. R. Reynolds, Macromolecules, 1999, 33, 4272-4278; (b) C. Gao and D. Yan, Macromolecules, 2001, 34, 156-161; (c) T. Emrick, H. T. Chang and J. M. J. Frechet, Macromolecules, 1999, 32, 63806382; (d) M. Jikei, S. H. Chon, M. Kakimoto, S. Kawauchi, T. Imase and J. Watanabe, Macromolecules, 1999, 32, 2061-2064; (e) S. Russo, A. Boulares, A. da Rin, A. Mariani and M. E. Cosulich, Macromol. Symp., 1999, 143, 309-321; (f) S. M. Ahoni, Polym. Adv. Technol., 1995, 6, 373; $(g)$ W. B. Wu, S. H. Ye, G. Yu, Y. Q. Liu, J. G. Qin and Z. Li, Macromol. Rapid Commun., 2012, 33, 164-171; (h) W. Wu, S. Ye, L. Huang, L. Xiao, Y. Fu, Q. Huang, G. Yu, Y. Liu, J. Qin, Q. Li and Z. Li, J. Mater. Chem., 2012, 22, 6374-6382.

4 J. M. J. Frechet, M. Henmi, I. Gitsov, S. Aoshima, M. Leduc and R. B. Grubbs, Science, 1995, 269, 1080-1083.

5 (a) C. J. Hawker, J. M. J. Frechet, R. B. Grubbs and J. Dao, J. Am. Chem. Soc., 1995, 117, 10763-10764; (b) S. G. Gaynor, S. Z. Edelman and K. Matyjaszewski, Macromolecules, 1996, 29, 1079-1081.

6 (a) A. Dworak, W. Walach and B. Trzebicka, Macromol. Chem. Phys., 1995, 196, 1963-1970; (b) M. Suzuki, S. Yoshida, K. Shiraga and T. Saegusa, Macromolecules, 1998, 31, 1716-1719; (c) H. Magnusson, E. Malmström and A. Hult, Macromol. Rapid Commun., 1999, 20, 453-457; (d) M. Bednarek, T. Biedron, J. Helinski, K. Kaluzynski, P. Kubisa and S. Penczek, Macromol. Rapid Commun., 1999, 20, 369-372; (e) A. Sunder, R. Hanselmann, H. Frey and R. Mülhaupt, Macromolecules, 1999, 32, 4240-4246.

7 (a) A. Sunder, J. Heinemann and H. Frey, Chem.-Eur. J., 2000, 6, 2499-2505; (b) B. Voit, C. R. Chim., 2003, 6, 821-832.

8 (a) J. Liu, J. W. Y. Lam and B. Z. Tang, Chem. Rev., 2009, 109, 57995867; (b) M. Häußler and B. Z. Tang, Adv. Polym. Sci., 2007, 209, 158; (c) R. Zheng, H. Dong, H. Peng, J. W. Y. Lam and B. Z. Tang, Macromolecules, 2004, 37, 5196-5210.

9 (a) J. Shi, C. K. W. Jim, F. Mahtab, J. Liu, J. W. Y. Lam, H. H. Y. Sung, I. D. Williams, Y. P. Dong and B. Z. Tang, Macromolecules, 2010, 43, 680-690; (b) J. Liu, R. Zheng, Y. Tang, M. Häußler, J. W. Y. Lam, A. Qin, M. Ye, Y. Hong, P. Gao and B. Z. Tang, Macromolecules, 2007, 40, 7473-7486; (c) M. Häußler, J. Liu, R. Zheng, J. W. Y. Lam, A. Qin and B. Z. Tang, Macromolecules, 2007, 40, 1914-1925; (d) Z. Li, A. Qin, J. W. Y. Lam, Y. Q. Dong, Y. P. Dong, C. Ye, I. D. Williams and B. Z. Tang, Macromolecules, 2006, 39, 1436-1442.

10 L. W. Tutt and A. Kost, Nature, 1992, 356, 225-226.
11 J. D. Luo, Z. L. Xie, J. W. Y. Lam, L. Cheng, H. Y. Chen, C. F. Qiu, H. S. Kwok, X. W. Zhan, Y. Q. Liu, D. B. Zhu and B. Z. Tang, Chem. Commun., 2001, 1740-1741.

12 (a) J. Liu, Y. Zhong, J. W. Y. Lam, P. Lu, Y. Hong, Y. Yu, Y. Yue, F. Mahtab, H. H. Y. Sung, I. Williams, K. S. Wong and B. Z. Tang, Macromolecules, 2010, 43, 4921-4936; (b) J. Liu, J. W. Y. Lam and B. Z. Tang, J. Inorg. Organomet. Polym. Mater., 2009, 19, 249-285.

13 (a) Y. Hong, J. W. Y. Lam and B. Z. Tang, Chem. Soc. Rev., 2011, 40, 5361-5388; (b) Y. Hong, J. W. Y. Lam and B. Z. Tang, Chem. Commun., 2009, 4332-4353; (c) J. Huang, X. Yang, J. Y. Wang, C. Zhong, L. Wang, J. G. Qin and Z. Li, J. Mater. Chem., 2012, 22, 2478-2484.

14 J. C. de Mello, H. F. Wittmann and R. H. Friend, Adv. Mater., 1997, 9, 230-232.

15 (a) P. Kundu, K. R. J. Thomas, J. T. Lin, Y. T. Tao and C. H. Chien, Adv. Funct. Mater., 2003, 13, 445-452; (b) J. Y. Shen, C. Y. Lee, T. H. Huang, J. T. Lin, Y. T. Tao, C. H. Chien and C. Tsai, J. Mater. Chem., 2005, 15, 2455-2463.

16 A. Qin, C. K. C. Jim, Y. Tang, J. W. Y. Lam, J. Liu, F. Mahtab, P. Gao and B. Z. Tang, J. Phys. Chem. B, 2008, 112, 9281-9288.

17 (a) C. W. Spangler, J. Mater. Chem., 1999, 9, 2013-2020; (b) R. C. Hollins, Curr. Opin. Solid State Mater. Sci., 1999, 4, 189-196.

18 (a) A. D. Slepkov, F. A. Hegmann, Y. Zhao, R. R. Tykwinski and K. Kamada, J. Chem. Phys., 2002, 116, 3834-3840; (b) A. D. Slepkov, F. A. Hegmann, S. Eisler, E. Elliott and R. R. Tykwinski, J. Chem. Phys., 2004, 120, 6807-6810; (c) S. Eisler, A. D. Slepkov, E. Elliott, T. Luu, R. McDonald, F. A. Hegmann and R. R. Tykwinski, J. Am. Chem. Soc., 2005, 127, 2666-2676; (d) I. Ledoux, I. D. W. Samuel, J. Zyss, S. N. Yaliraki, F. J. Schattenmann, R. R. Schrock and R. J. Silbey, Chem. Phys., 1999, 245, 1-3; (e) H. Peng, L. Cheng, J. Luo, K. Xu, Q. Sun, Y. Dong, F. Salhi, P. P. S. Lee, J. Chen and B. Z. Tang, Macromolecules, 2002, 35, 5349-5351.

19 (a) S. W. Thomas, G. D. Joly and T. M. Swager, Chem. Rev., 2007, 107, 1339-1386; (b) U. H. F. Bunz, Chem. Rev., 2000, 100, 16051644; (c) J. Liu, Y. Zhong, P. Lu, Y. Hong, J. W. Y. Lam, F. Mahtab, Y. Yu, K. S. Wong and B. Z. Tang, Polym. Chem., 2010, 1, 426-429; (d) J. S. Yang and T. M. Swager, J. Am. Chem. Soc., 1998, 120, 11864-11873; (e) S. J. Toal and W. C. Trogler, J. Mater. Chem., 2006, 16, 2871-2883; ( $f$ ) Q. Zhou and T. M. Swager, J. Am. Chem. Soc., 1995, 117, 12593-12602; (g) H. Nie, Y. Zhao, M. Zhang, Y. Ma, M. Baumgarten and K. Mullen, Chem. Commun., 2011, 47, 1234-1236; $(h)$ P. G. Del Rosso, M. F. Almassio and R. O. Garay, Tetrahedron Lett., 2011, 52, 4911-4915; (i) P. Lu, J. W. Y. Lam, J. Liu, C. K. W. Jim, W. Yuan, N. Xie, Y. Zhong, Q. Hu, K. S. Wong, K. K. L. Cheuk and B. Z. Tang, Macromol. Rapid Commun., 2010, 31, 834-839.

20 (a) J. C. Sanchez, A. G. Dipasquale, A. L. Rheingold and W. C. Trogler, Chem. Mater., 2007, 19, 6459-6470; (b) D. Zhao and T. M. Swager, Macromolecules, 2005, 38, 9377-9384; (c) J. C. Sanchez and W. C. Trogler, J. Mater. Chem., 2008, 18, 31433156; (d) T. Naddo, Y. Che, W. Zhang, K. Balakrishnan, X. Yang, M. Yen, J. Zhao, J. S. Moore and L. Zhang, J. Am. Chem. Soc., 2007, 129, 6978-6979; (e) Y. Long, H. Chen, Y. Yang, H. Wang, Y. Yang, N. Li, K. Li, J. Pei and F. Liu, Macromolecules, 2009, 42, 6501-6509.

21 H. Sohn, M. J. Sailor, D. Magde and W. C. Trogler, J. Am. Chem. Soc., 2003, 125, 3821-3830. 